\title{
3D modeling of electric fields in the LUX detector
}

The LUX collaboration: D.S. Akerib, ${ }^{a, b, c}$ S. Alsum, ${ }^{d}$ H.M. Araújo, ${ }^{e}$ X. Bai, ${ }^{f}$ A.J. Bailey, ${ }^{e}$ J. Balajthy, ${ }^{g}$ P. Beltrame, ${ }^{h}$ E.P. Bernard,${ }^{i, j}$ A. Bernstein, ${ }^{k}$ T.P. Biesiadzinski, ${ }^{a, b, c}$ E.M. Boulton, ${ }^{i, j}$ P. Brás, ${ }^{l}$ D. Byram,${ }^{m, n}$ S.B. Cahn, ${ }^{j}$ M.C. Carmona-Benitez, ${ }^{o, p}$ C. Chan, ${ }^{q}$ A. Currie, ${ }^{e}$ J.E. Cutter, ${ }^{r}$ T.J.R. Davison, ${ }^{h}$ A. Dobi, ${ }^{s}$ E. Druszkiewicz, ${ }^{t}$ B.N. Edwards,${ }^{j}$ S.R. Fallon, ${ }^{u}$ A. Fan, ${ }^{b, c}$ S. Fiorucci, ${ }^{q, s}$ R.J. Gaitskell, ${ }^{q}$ J. Genovesi, ${ }^{,}$C. Ghag, ${ }^{v}$ M.G.D. Gilchriese, ${ }^{s}$ C.R. Hall, ${ }^{g}$ M. Hanhardt, ${ }^{f, n}$ S.J. Haselschwardt, ${ }^{p}$ S.A. Hertel,,${ }^{j, s, w}$ D.P. Hogan, ${ }^{i}$ M. Horn, ${ }^{i, j, n}$ D.Q. Huang, ${ }^{q}$ C.M. Ignarra, ${ }^{b, c}$ R.G. Jacobsen, ${ }^{i}$ W. Ji $,{ }^{a}, b, c$ K. Kamdin, ${ }^{i}$ K. Kazkaz, ${ }^{k}$ D. Khaitan, ${ }^{t}{ }^{R}$. Knoche, ${ }^{g}$ N.A. Larsen, ${ }^{j}$ B.G. Lenardo, ${ }^{k, r}$ K.T. Lesko, ${ }^{s}$ A. Lindote, ${ }^{l}$ M.I. Lopes, ${ }^{l}$ A. Manalaysay, ${ }^{r}$ R.L. Mannino, ${ }^{d, x}$ M.F. Marzioni, ${ }^{h}$ D.N. McKinsey, ${ }^{i, j, s}$ D.-M. Mei, ${ }^{m}$ J. Mock, ${ }^{u}$ M. Moongweluwan, ${ }^{t}$ J.A. Morad, ${ }^{r}$ A.St.J. Murphy, ${ }^{h}$ C. Nehrkorn, ${ }^{p}$ H.N. Nelson, ${ }^{p}$ F. Neves, ${ }^{l}$ K. O'Sullivan, ${ }^{i, j, s}$ K.C. Oliver-Mallory, ${ }^{i}$ K.J. Palladino, ${ }^{b, c, d}$ E.K. Pease,${ }^{i, j}$ C. Rhyne, ${ }^{q}$ S. Shaw, ${ }^{p, v}$ T.A. Shutt, ${ }^{a, c}$ C. Silva, ${ }^{l}$ M. Solmaz, ${ }^{p}$ V.N. Solovov, ${ }^{l}$ P. Sorensen, ${ }^{s}$ T.J. Sumner, ${ }^{e}$ M. Szydagis, ${ }^{u}$ D.J. Taylor, ${ }^{n}$ W.C. Taylor, ${ }^{q}$ B.P. Tennyson, ${ }^{j}$ P.A. Terman, ${ }^{x}$ D.R. Tiedt, ${ }^{f}$ W.H. To,${ }^{b, c, y}$ M. Tripathi, ${ }^{r}$ L. Tvrznikova, ${ }^{i, j, s, 1}$ S. Uvarov, ${ }^{r}$ V. Velan, ${ }^{i}$ J.R. Verbus, ${ }^{q}$ R.C. Webb, ${ }^{x}$ J.T. White, ${ }^{x}$ T.J. Whitis, ${ }^{a}, b, c$ M.S. Witherell, ${ }^{s}$ F.L.H. Wolfs, ${ }^{t}$ J. Xu, ${ }^{k}$ K. Yazdani, ${ }^{e}$ S.K. Young, ${ }^{u}$ C. Zhang. ${ }^{m}$

${ }^{a}$ Case Western Reserve University, Department of Physics, 10900 Euclid Ave, Cleveland, OH 44106, USA

${ }^{b}$ SLAC National Accelerator Laboratory, 2575 Sand Hill Road, Menlo Park, CA 94205, USA

${ }^{c}$ Kavli Institute for Particle Astrophysics and Cosmology, Stanford University, 452 Lomita Mall, Stanford, CA 94309, USA

${ }^{d}$ University of Wisconsin-Madison, Department of Physics, 1150 University Ave., Madison, WI 53706, USA ${ }^{e}$ Imperial College London, High Energy Physics, Blackett Laboratory, London SW7 2BZ, United Kingdom ${ }^{f}$ South Dakota School of Mines and Technology, 501 East St Joseph St., Rapid City, SD 57701, USA Imperial College London, High Energy Physics, Blackett Laboratory, London SW7 2BZ, United Kingdom ${ }^{g}$ University of Maryland, Department of Physics, College Park, MD 20742, USA

${ }^{h}$ SUPA, School of Physics and Astronomy, University of Edinburgh, Edinburgh EH9 3FD, United Kingdom ${ }^{i}$ University of California Berkeley, Department of Physics, Berkeley, CA 94720, USA

${ }^{j}$ Yale University, Department of Physics, 217 Prospect St., New Haven, CT 06511, USA

${ }^{k}$ Lawrence Livermore National Laboratory, 7000 East Ave., Livermore, CA 94551, USA

${ }^{l}$ LIP-Coimbra, Department of Physics, University of Coimbra, Rua Larga, 3004-516 Coimbra, Portugal

${ }^{m}$ University of South Dakota, Department of Physics, 414E Clark St., Vermillion, SD 57069, USA

${ }^{n}$ South Dakota Science and Technology Authority, Sanford Underground Research Facility, Lead, SD 57754, USA

${ }^{o}$ Pennsylvania State University, Department of Physics, 104 Davey Lab, University Park, PA 16802-6300, USA

\footnotetext{
${ }^{1}$ Corresponding author
} 
${ }^{p}$ University of California Santa Barbara, Department of Physics, Santa Barbara, CA 93106, USA

${ }^{q}$ Brown University, Department of Physics, 182 Hope St., Providence, RI 02912, USA

${ }^{r}$ University of California Davis, Department of Physics, One Shields Ave., Davis, CA 95616, USA

${ }^{s}$ Lawrence Berkeley National Laboratory, 1 Cyclotron Rd., Berkeley, CA 94720, USA

${ }^{t}$ University of Rochester, Department of Physics and Astronomy, Rochester, NY 14627, USA

${ }^{u}$ University at Albany, State University of New York, Department of Physics, 1400 Washington Ave., Albany, NY 12222, USA

${ }^{v}$ Department of Physics and Astronomy, University College London, Gower Street, London WCIE 6BT, United Kingdom

${ }^{w}$ University of Massachusetts, Amherst Center for Fundamental Interactions and Department of Physics, Amherst, MA 01003-9337 USA

${ }^{x}$ Texas A \& M University, Department of Physics, College Station, TX 77843, USA

${ }^{y}$ California State University Stanislaus, Department of Physics, 1 University Circle, Turlock, CA 95382, USA

E-mail: lucie.tvrznikova@yale.edu

Abstract: This work details the development of a three-dimensional (3D) electric field model for the LUX detector. The detector took data to search for weakly interacting massive particles (WIMPs) during two periods. After the first period completed, a time-varying non-uniform negative charge developed in the polytetrafluoroethylene (PTFE) panels that define the radial boundary of the detector's active volume. This caused electric field variations in the detector in time, depth and azimuth, generating an electrostatic radially-inward force on electrons on their way upward to the liquid surface. To map this behavior, 3D electric field maps of the detector's active volume were generated on a monthly basis. This was done by fitting a model built in COMSOL Multiphysics to the uniformly distributed calibration data that were collected on a regular basis. The modeled average PTFE charge density increased over the course of the exposure from -3.6 to $-5.5 \mu \mathrm{C} / \mathrm{m}^{2}$. From our studies, we deduce that the electric field magnitude varied locally while the mean value of the field of $\sim 200 \mathrm{~V} / \mathrm{cm}$ remained constant throughout the exposure. As a result of this work the varying electric fields and their impact on event reconstruction and discrimination were successfully modeled.

Keywords: Analysis and statistical methods; Detector modelling and simulations II (electric fields, charge transport, multiplication and induction, pulse formation, electron emission, etc); Noble liquid detectors (scintillation, ionization, double-phase); Dark Matter detectors (WIMPs, axions, etc.)

ArXiv EPrint: 1709.00095 


\section{Contents}

1 The LUX detector 1

2 Modeling electric fields inside the LUX detector 3

2.1 LUX 3D model geometry 3

2.2 Electric fields modeling 4

2.3 Modeling charges in the LUX detector $\quad 6$

2.4 Alternative explanations for the field distortion 8

3 Modeling detector observables 9

3.1 WS2013 modeling results 9

3.2 Alternative determination of electric field magnitude using NEST 11

4 Time-dependence of electric fields during WS2014-16 13

$\begin{array}{ll}4.1 \text { Charge evolution } & 13\end{array}$

$\begin{array}{lll}4.2 & \text { Electric fields in WS2014-16 analysis } & 14\end{array}$

$\begin{array}{lll}5 & \text { Summary } & 17\end{array}$

\section{The LUX detector}

The Large Underground Xenon (LUX) experiment is a two-phase (liquid-gas) xenon time projection chamber (TPC) searching for weakly interacting massive particles, a favored dark matter candidate. The detector uses a dodecagonal prism shaped active volume bounded in $z$ by cathode and gate wire grids and in $(x, y)$ by 12 polytetrafluoroethylene (PTFE) panels and contains $251 \mathrm{~kg}$ of ultra pure xenon. The inner cryostat is $101 \mathrm{~cm}$ tall and $62 \mathrm{~cm}$ in diameter. The active volume diameter, as measured at $-100^{\circ} \mathrm{C}$ between parallel opposite faces, is $47.3 \mathrm{~cm}$. The distance between cathode and gate grids is $48.3 \mathrm{~cm}$ and distance between gate and anode grids is $1 \mathrm{~cm}$.

Energy deposited by particle interactions induces two measurable signals in the active volume: a prompt scintillation of vacuum ultraviolet (VUV) photons (S1) and an ionization signal from the liberated electrons (S2). The S1 photons are emitted at the interaction site and are detected by top and bottom arrays of 61 photomultiplier tubes (PMTs) each. The detector's drift field pulls free electrons to the liquid surface where they are extracted into the gas, producing a secondary electroluminescence S2 signal on their path to the anode. The S2 photons are detected by both PMT arrays with localization of the signal in the top array. The PMT signals from both light pulses, S1 and S2, along with their time separation that defines the depth of the interaction, allow the reconstruction of interaction vertices in three dimensions $\left(x_{S 2}, y_{S 2}, t\right)$. The ratio of free charge to scintillation light, typically expressed as $\log _{10}(\mathrm{~S} 2 / \mathrm{S} 1)$, is dependent on the electric field at the event site and is used to distinguish electronic recoils (ERs) and nuclear recoil (NRs) produced by 
incoming particles interacting with xenon atoms. The NR signals exhibit less variation as a function of field in comparison to ERs, due to the lower ionization of NRs and thus lower importance of electron-ion recombination. This enables discrimination between ER and NR events and makes liquid xenon TPC detectors viable dark matter discovery experiments. Leveraging these capabilities helped LUX produce world-leading exclusion limits for both spin-independent and spin-dependent WIMP-nucleon scattering cross-sections, axion searches, and pioneer many innovative calibration techniques [1-6]. Further technical details about the LUX detector can be found in [7].

The detector took WIMP search data during two periods; from April 21 to August 8, 2013 (WS2013) and from September 11, 2014 until May 2, 2016 (WS2014-16). Between WS2013 and WS2014-16, from January to March 2014, the detector grids were conditioned to improve the voltages at which the electrodes could be biased. Afterward, the detector was kept at room temperature for one month in April 2014 before starting WS2014-16. During the conditioning, potentials were held just above the onset of sustained discharge and maintained for many days, akin to the burn-in period often employed in room-temperature proportional counter commissioning [811]. First, the potentials on gate and anode grids were raised to $-5 \mathrm{kV}$ and $7 \mathrm{kV}$, respectively, while in cold xenon gas $(180 \mathrm{~K})$. Subsequently, the potential on the cathode was raised to $-20 \mathrm{kV}$ while in xenon gas at room temperature $(296 \mathrm{~K})$. As a result of conditioning, a higher applied anode voltage enabled increased electron extraction efficiency (i.e. the fraction of electrons which promptly cross the liquid-gas interface) from $49 \pm 3 \%$ in WS2013 to $73 \pm 4 \%$ in WS2014-16 [4]. After the conditioning campaign, ${ }^{83 \mathrm{~m}} \mathrm{Kr}$ calibration data (see Section 2.2) revealed that the electron drift trajectories were significantly altered from the regular near-vertical paths observed in WS2013. figure 1 shows the reconstructed radius and drift time of ${ }^{83 \mathrm{~m}} \mathrm{Kr}$ calibration events next to the PTFE wall, before and after conditioning. A strong bias in azimuthal angle and $z$ was observed, and seen to change throughout WS2014-16. The contours were created by finding the average number of events in all the non-empty bins of a $2 \mathrm{D}$ histogram in $\left(r^{2} \mathrm{~s} 2, t\right)$ and then drawing a contour through those bins that contained half of the average number of events. Therefore the contours approximate the edges of the detector's active volume.

We attribute the time evolution of the electric field to the migration of negative charges (electrons) and positive charges (holes) in the PTFE panels that define the radial boundary of the active volume and serve as a reflector to VUV scintillation photons [12]. Discharges in the xenon gas during conditioning created VUV photons with energies greater than the band gap of PTFE [13-17], which in turn created electron-hole pairs within $1-2 \mu \mathrm{m}$ of the PTFE panels [18]. The behavior of charges in PTFE and other Teflon materials has been widely studied at room and elevated temperatures [19-22], but not at liquid xenon (LXe) temperatures. The energy levels of the electron and hole traps have been measured to be $0.85-1.0 \mathrm{eV}$ and $0.80-0.90 \mathrm{eV}$ respectively, showing a slightly higher energy trap for electrons [18]. PTFE is known to be a strong insulator with dark-current resistivity of $\rho \sim 3 \times 10^{20} \Omega \cdot \mathrm{cm}$ measured at $5 \times 10^{-6}$ torr at $25^{\circ} \mathrm{C}$ [20] and is able to maintain negative charge densities of at least $0.1 \mathrm{mC} / \mathrm{m}^{2}$ [23]. This results in a faster removal of holes compared to electrons under the applied field creating the observed abundance of negative charges in the PTFE panels. Furthermore, this behavior varies over time thus introducing a strong radially-inward drift of electrons during WS2014-16 [4] varying in time, depth and azimuth as described in section 4.1 .

Knowledge of the electric field inside the detector is necessary for $(x, y)$ position reconstruction 


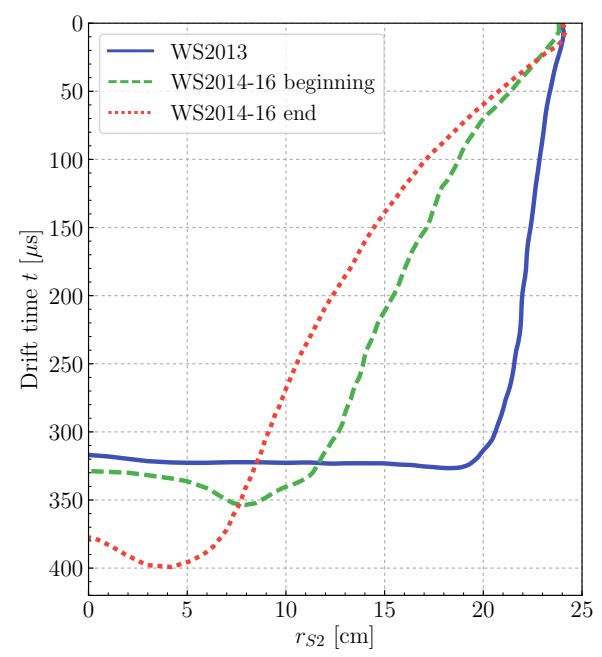

Figure 1. Contour plot illustrating detectors edges from the reconstructed positions of the ${ }^{83 \mathrm{~m}} \mathrm{Kr}$ calibration data. Differences between WS2013 (data from 2013-05-10, solid blue) and the time-dependent WS2014-16 (data from 2014-09-03, dashed green and 2016-05-03, dotted red) are clearly seen. Due to the changing electric fields, ${ }^{83 \mathrm{~m}} \mathrm{Kr}$ calibration data from the later science run had events with greater drift times.

since the drift path of the S2 electrons is field-dependent. Event reconstruction is also fielddependent due to the effect of the drift field on recombination. Since the conditioning altered the electric fields inside the detector in a non-symmetric manner, a full 3D model of the fields was needed throughout the 23 month-long WS2014-16. This paper describes the method used to develop these field models.

\section{Modeling electric fields inside the LUX detector}

Electric field models were developed both with and without charge densities in the PTFE panels to understand the detector behavior. Prior to developing a model for the changing electric fields during WS2014-16, a 3D model was developed without static charges in the PTFE panels to confirm our understanding of the calibration data from WS2013. The 3D model was built using the AC/DC Module of COMSOL Multiphysics v5.0 ${ }^{\circledR}$ [24], a commercially available finite element simulation software.

\subsection{LUX 3D model geometry}

A full 3D model was needed due to the detector's geometry. The active volume takes a shape of a regular dodecagonal prism as shown in the left panel of figure 2 and the five detector grids (bottom shield, cathode, gate, anode, top shield) are rotated by $60^{\circ}$ with respect to each other, leaving a geometry that is not azimuthally or otherwise symmetric. The $2 \mathrm{D}$ cross-section of the model used in the 3D simulations is shown in the right panel of figure 2; the figure also illustrates some of the adopted simplifications. Due to the high degree of complexity of the detector, only the active region of the detector was modeled in detail; the bottom shield and anode grids were modeled as planes imposing electrostatic boundaries for the height of the active region of the detector. 


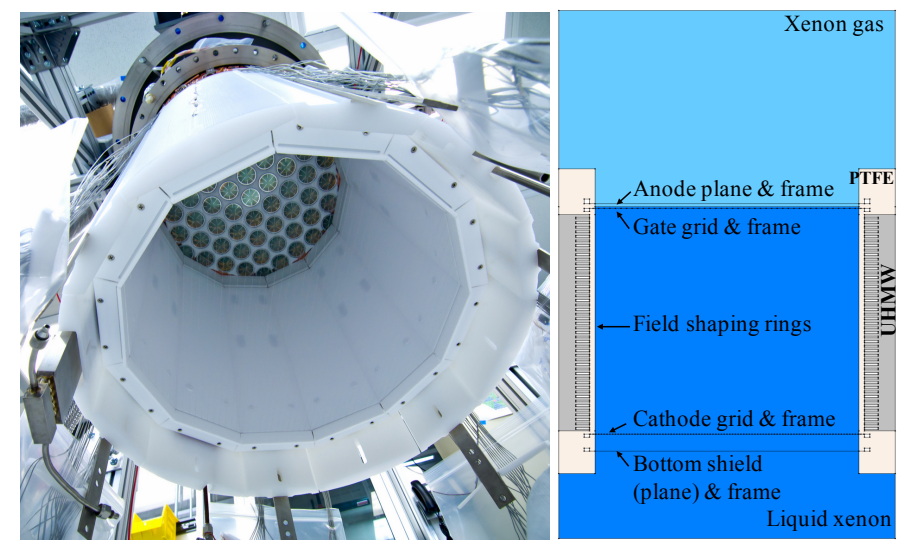

Figure 2. Left: bottom view of the LUX detector showing the regular dodecagonal prism shape, the PTFE panels, and the top PMT array. Right: cross-section of the 3D LUX COMSOL model.

Since the model spans 5 orders of magnitude in length scale (from $28 \mu \mathrm{m}$ for the grid wire diameter to $1.1 \mathrm{~m}$ for the inner vacuum cryostat height), the cathode and gate grid wires were modeled as parallel lines with zero diameter to accelerate the convergence of the model. As with all simplifications, test cases were studied to ensure this wire diameter change had a negligible effect on the resulting solution without a loss of field resolution [25]. Further details about the detector grids along with applied grid voltages from WS2013 and WS2014-16 are summarized in Table 1. The details of the ultra-high-molecular-weight polyethylene (UHMW) and PTFE structures surrounding the active volume were simplified and large metal objects residing outside of the field cage (i.e. cathode high voltage cable, heat exchanger) were omitted since a simulation confirmed they do not affect the electric field within the active volume. The relative DC dielectric constants of the materials used in the model were: $\varepsilon_{\text {liquid xenon }}=1.95, \varepsilon_{\text {gaseous xenon }}=1.0, \varepsilon_{P T F E}=2.1$, $\varepsilon_{U H M W}=2.3$.

\subsection{Electric fields modeling}

Once the detector geometry, materials, electrical dielectric properties, and voltages were assigned to detector volumes and boundaries, this defined the electrostatic conditions. COMSOL then generated a mesh by discretizing the space into tetrahedra and a proposed field map was generated. Using an adaptive mesh refinement allowed further improvement of mesh quality while minimizing solution error. This process is illustrated in figure 3.

The resulting electric field values found in COMSOL Multiphysics were exported and used to produce a simulation dataset of electron-like particles in Python v2.7. This was accomplished by starting electron-like test particles uniformly throughout the detector's active volume and recording their intersection with the liquid surface after drifting. These particles were propagated step-wise in $1-3 \mu$ s intervals using interpolated velocity since the electron drift velocity in LXe varies with electric field [26].

Once the electron-like test particles reached the liquid surface, the simulated drift time $\left(t_{\text {sim }}\right)$ and location of the simulated S2 light production $\left(x_{S 2_{\text {sim }}}, y_{S 2_{\text {sim }}}\right)$ were compared with the $\left(x_{S 2}, y_{S 2}, t\right)$ 
Table 1. Grid properties and voltages as relevant to the construction of the electric field model used in WS2013 and WS2014-16 simulations, including description of geometric simplifications.

\begin{tabular}{|lccccc|}
\hline Grid & $\begin{array}{c}\text { Bottom } \\
\text { shield }\end{array}$ & Cathode & Gate & Anode & $\begin{array}{c}\text { Top } \\
\text { shield }\end{array}$ \\
\hline$z^{\dagger}[\mathrm{cm}]$ & 2.0 & 5.6 & 53.9 & 54.9 & 58.6 \\
Wire $\varnothing[\mu \mathrm{m}]$ & 206.0 & 206.0 & 101.6 & 28.4 & 50.8 \\
Pitch [mm] & 10.00 & 5.00 & 5.00 & 0.25 & 5.00 \\
Angle [deg] & 15 & 75 & 15 & N/A & 135 \\
Modeled as & Plane & $\varnothing 0$ wires & $\varnothing 0$ wires & Plane & Absent \\
WS2013 [kV] & -2 & -10 & -1.5 & 3.5 & -1 \\
WS2014-16 $[\mathrm{kV}]$ & -2 & -8.5 & 1 & 7 & -1 \\
\hline
\end{tabular}

${ }^{\dagger} z$ is defined as vertical distance from the face of the bottom PMT array, accounting for thermal contraction as appropriate.
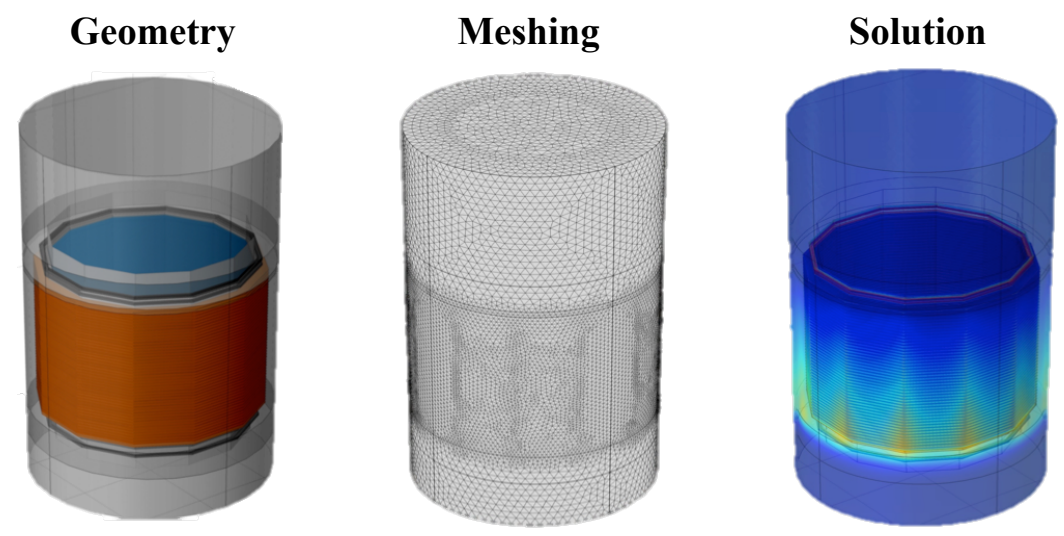

Figure 3. Steps in building the 3D model of the electric fields in the LUX detector using COMSOL Multiphysics v5.0.

distribution of ${ }^{83 \mathrm{~m}} \mathrm{Kr}$ calibration data. See section 2.3 for details.

${ }^{83 \mathrm{~m}} \mathrm{Kr}$ was injected weekly into the detector as a calibration source producing $\sim 10^{6}$ events that distribute homogeneously in the detector's active volume within minutes. This makes it a perfect source to use in developing 3D maps of the electric field. The radioactive isotope ${ }^{83 \mathrm{~m}} \mathrm{Kr}$ has a half-life of 1.83 hours. The decay occurs in two transitions of 32.1 and $9.4 \mathrm{keV}$ respectively, with an intervening half-life of $154 \mathrm{~ns}$. These two transitions can each proceed according to multiple decay channels, with a high probability of internal conversion followed by Auger emission, resulting in a high concentration of decay energy into electron modes. More details regarding ${ }^{83 \mathrm{~m}} \mathrm{Kr}$ calibration of the LUX detector can be found in [27]. 


\subsection{Modeling charges in the LUX detector}

A large change in the detector's electric field occurred between WS2013 and WS2014-16 which affected electron paths as shown in figure 1 . This change in field can be modeled with negative charge densities present in the PTFE panels; the consequence of this accumulated charge is illustrated in figure 4 .
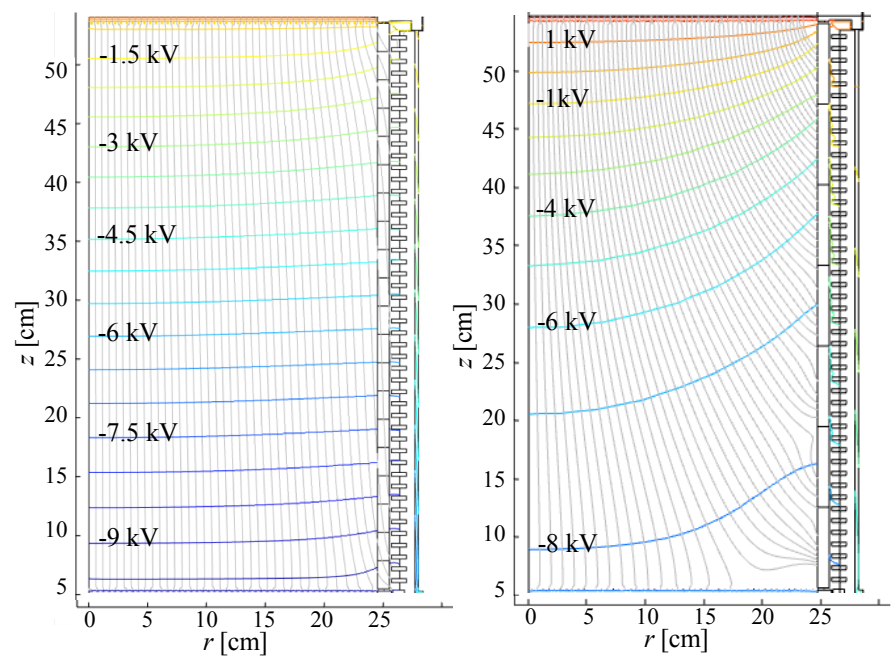

Figure 4. Two simple axially symmetric COMSOL Multiphysics models built for visualization purposes. Left: electric field lines and equipotentials in the LUX detector during WS2013. A radially-inward component is seen, resulting from the geometry of the field cage and the grids. Right: electric field lines implied by ${ }^{83 \mathrm{~m}} \mathrm{Kr}$ from $2015-04-26$ showing the much stronger radially-inward component modeled by negative charge densities in the PTFE panels.

To gain intuition about the effects on electron paths caused by charge in the PTFE panels, a simple simulation was performed by depositing $-1 \mu \mathrm{C} / \mathrm{m}^{2}$ of charge density in either the lower, middle, or upper third of a PTFE panel; the resulting force causing inward displacement of events that originate near or below the charged site is demonstrated in figure 5 .

To model the detector behavior in WS2014-16 first the basic charge-free model was modified to include WS2014-16 grid voltages. Then 42 additional COMSOL Multiphysics models were built with voltages on all boundaries set to $0 \mathrm{~V}$. The 12 PTFE panels surrounding the active volume were split into 42 tiles by defining 6 angular and 7 vertical divisions of equal width and height. Therefore there were 2 PTFE panels in each angular division. Each one of the 42 models included $-1 \mu \mathrm{C} / \mathrm{m}^{2}$ of charge on one of the 42 tiles. These 43 datasets (arising from 42 models with charge and one without charge) served as basis vectors since the charge on any one tile could be scaled up or down as needed. Leveraging the superposition principle, these models linearly combined to produce electric field maps of arbitrary charge distribution. Those models were solved by COMSOL Multiphysics and the resulting 43 electric field vector maps were exported on a $1 \mathrm{~cm}^{3}$ grid to a Python script where all further calculations were performed. For a given hypothetical charge distribution in the PTFE panels a simulation dataset was made and compared to ${ }^{83 \mathrm{~m}} \mathrm{Kr}$ calibration data. This charge was varied with an iterative algorithm until the distributions converged on a stable solution. 


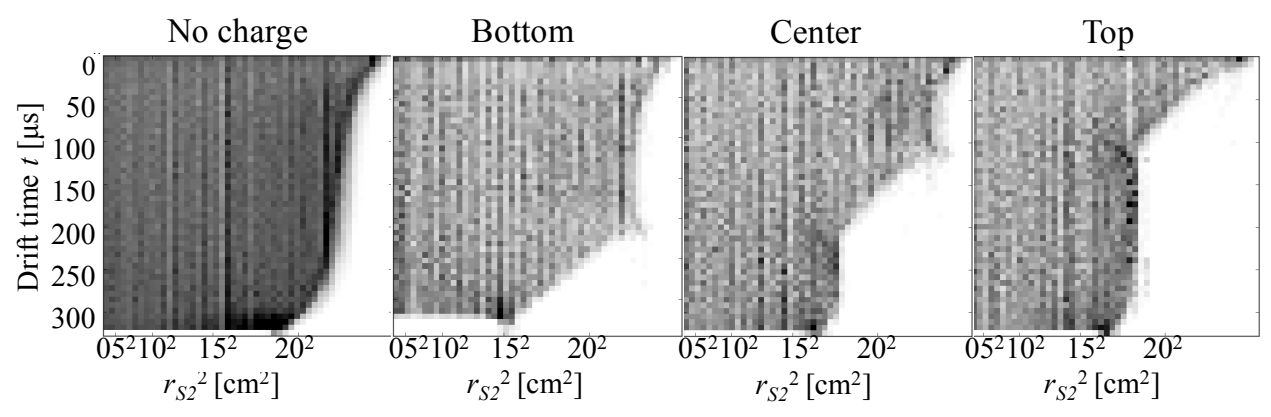

Figure 5. Effect of negative charge densities in the PTFE panels on events in the detector. The 4 figures illustrate COMSOL models with no charge in the PTFE panels and with $-1 \mu \mathrm{C} / \mathrm{m}^{2}$ deposited at the lower, middle, and upper segments of a PTFE panel causing a strong inward push for events that originate near or below the charge site.

Unphysical behavior emerged when 2 neighboring vertical tiles contained very different charges. To mitigate these sharp discontinuities the charge on each tile was set to fall off linearly both in the azimuthal and in $z$ directions. This smoothing was defined such that in the azimuthal direction each tile had a uniform $-1 \mu \mathrm{C} / \mathrm{m}^{2}$ charge density from $1 / 4$ to $3 / 4$ of tile width. This charge density then decreased linearly to $0 \mu \mathrm{C} / \mathrm{m}^{2}$ through $1 / 4$ of the width of the neighboring tile. Therefore the charge density in the azimuthal direction was continuous. The same technique was applied in the $z$ direction except that the bottommost and topmost edge tiles were smoothed in only the direction pointing toward the center of the panel.

After the simulated particles were drifted, a likelihood function was used to compare ${ }^{83 \mathrm{~m}} \mathrm{Kr}$ data to simulation. Then the initial charge hypothesis was refined and this approach was iterated using the Metropolis-Hastings algorithm until convergence of results was seen. The MetropolisHastings algorithm is a Markov Chain Monte Carlo method for sampling from a multi-dimensional distribution with a high number of degrees of freedom. First an initial guess was made for the amount of charge on each tile and the corresponding model was created. Each subsequent guess was randomly sampled from a Gaussian distribution centered at each charge density with a width of $\sim 0.1 \mu \mathrm{C} / \mathrm{m}^{2}$. The ideal step size was tuned to achieve acceptance of new charge distributions of $\sim 23 \%$ [28], where the acceptance ratio was defined as

$$
\alpha=\frac{\mathcal{L}_{\text {new }}}{\mathcal{L}_{\text {old }}} .
$$

Here, $\mathcal{L}_{\text {new }}$ and $\mathcal{L}_{\text {old }}$ are the $\log$ likelihood functions for the current and previous steps, respectively. To speed up the convergence at the beginning of the modeling efforts, the amount of charge in the PTFE panels was adjusted manually to smooth out charge deviations between neighboring vertical panels before using the Metropolis-Hastings algorithm. As the dataset neared convergence a modified Metropolis-Hastings algorithm was used due to the high number of degrees of freedom (42). Instead of varying all charges simultaneously, as is usual for the algorithm, only one random charge was varied at a time which sped up convergence.

Once the final charge distribution was found, the information included in the resulting field maps was provided on a $1 \mathrm{~cm}^{3}$ grid that included the detector real space coordinates $(x, y, z)$, the electric 
field in each location, and the coordinates as seen by the detector $\left(x_{S 2}, y_{S 2}, t\right)$. The transformation between real space and the reconstructed S2 space can be seen in the two plots in figure 6 . The field maps were used in a Geant4-based LUXSim [29] simulation, position reconstruction, and limits code.
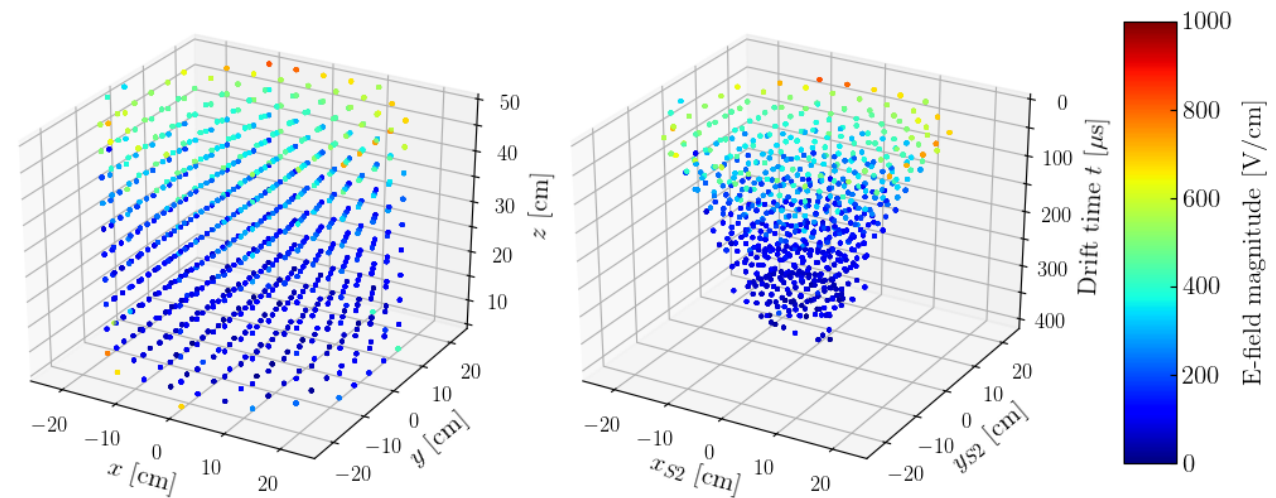

Figure 6. Transformation from the reconstructed S2 space (right) to real space (left). The color scale shows the electric field strength at each point using ${ }^{83 \mathrm{~m}} \mathrm{Kr}$ data from $2015-08-26$.

\subsection{Alternative explanations for the field distortion}

Other than the charge evolution in the PTFE surface, there are other plausible explanations for distorted fields inside a TPC. One possibility would be that drifting electrons land on the PTFE surface and do not get pulled toward the surface due to the high resistivity of PTFE. This is unlikely since the detector is designed to pull electrons up and away from the PTFE surface. Other explanations might be a short or open circuit in the field-shaping ring resistor chain. The field distortion caused by a shorted resistor was simulated in a simplified axially symmetric COMSOL model. A shorted resistor produces a small, very localized field deformity, unlike the detector-wide field distortion observed in WS2014-16. This effect would also not evolve throughout the detector operation.

An open circuit was also simulated in the axially symmetric COMSOL model. A field distortion is obvious and not unsimilar to the field observed in WS2014-16, however depending on the location of the open field, it can create large, dead, field-less regions in the detector. The resistance of the field-shaping ring resistor chain was measured before WS2013 and then again before WS2014-16. No significant change was observed, ruling out this explanation. An open circuit effect is also ruled out because it would not create a time-dependent component. Additionally, ${ }^{137} \mathrm{Cs}$ calibrations were performed in WS2014-16 using acrylic radioactive-source deployment tubes suspended along the side of the detector [7]. These calibrations showed events along the entire detector height, inconsistent with the presence of field-less regions. 


\section{Modeling detector observables}

To model the electric field, the simulation results were compared to the field implied by ${ }^{83 \mathrm{~m}} \mathrm{Kr}$ calibration data to ensure its accuracy. This comparison had to be done many times during the Metropolis Hastings algorithm rendering the traditional method of drifting electrons through the detector computationally too intensive and an alternative method comparing event densities was developed.

To begin, the ${ }^{83 \mathrm{~m}} \mathrm{Kr}$ events as seen in $\mathrm{S} 2$ space were reverse propagated through the modeled drift field to find the initial homogeneous distribution. In practice, this was accomplished by segmenting the ${ }^{83 \mathrm{~m}} \mathrm{Kr}$ data into tetrahedra within the active volume using Delaunay triangulation and recording the number of events enclosed by each segment. The vertices of those tetrahedra were drifted "back in time" through the electric field; each vertex started at the liquid surface and was drifted through the active volume, making the transformation from S2 to real space. The new volume of each tetrahedron after drifting, $V_{\text {tet }}$, was calculated and the number of ${ }^{83 \mathrm{~m}} \mathrm{Kr}$ events, $n_{S 2}$, contained in each tetrahedra recorded. Since in real space ${ }^{83 \mathrm{~m}} \mathrm{Kr}$ events were uniformly distributed throughout the detector, the number of events in each tetrahedron was proportional to its volume $n_{\text {real }}=N_{\mathrm{Kr}} * \frac{V_{\text {tet }}}{V_{\text {TPC }}}$, where $N_{\mathrm{Kr}}$ is the total number of ${ }^{83 \mathrm{~m}} \mathrm{Kr}$ events in the detector and $V_{\mathrm{TPC}}$ is the active volume of the detector. If the simulated electric field captured the field in the detector well, then $n_{\text {real }}$ and $n_{S 2}$ had equal number of events. The density comparison between ${ }^{83 \mathrm{~m}} \mathrm{Kr}$ data and simulation is illustrated in the right panel of figure 7.

To complete the fine-tuning of the model, the edges of the ${ }^{83 \mathrm{~m}} \mathrm{Kr}$ distribution were fully simulated to compare with data, in a method independent of the ${ }^{83 \mathrm{~m}} \mathrm{Kr}$ homogeneity in the detector. When approaching the final convergence of data and simulation only the edges of the ${ }^{83 \mathrm{~m}} \mathrm{Kr}$ distribution were used to fine tune the model. The detector was split into 12 angular sections corresponding to the 12 PTFE panels and negative charges were drifted to the liquid surface starting along the center of each panel face. An offset radius (set at $r=22 \mathrm{~cm}$ ) was used due to a higher fidelity of field maps slightly inward from the PTFE panels. The resulting simulated edge at the detector wall $(r=23.7 \mathrm{~cm})$ agrees with the edge found both from the ${ }^{83 \mathrm{~m}} \mathrm{Kr}$ data and from the location of the wall as determined from ${ }^{210} \mathrm{Po}$ background [30]. It is worth noting that even though only the detector wall was fitted, as shown in the left panel of figure 7 , the result correctly described the density distribution inside the entire detector.

\subsection{WS2013 modeling results}

A comparison of the 3D field model and ${ }^{83 \mathrm{~m}} \mathrm{Kr}$ data from WS2013 is shown in the left panel of figure 8. Excellent agreement is seen, without a need to tune any aspect of the model to improve agreement with the data. The value of the mostly uniform electric field inside the fiducial volume during WS2013 was $177 \pm 14 \mathrm{~V} / \mathrm{cm}$ as simulated in COMSOL Multiphysics. In WS2013 the fiducial volume was defined by drift time range of $38-305 \mu$ s and radius $r \leq 20 \mathrm{~cm}$, and contained $118 \mathrm{~kg}$ of LXe [2]. The electric field strength in WS2013 is illustrated in the right panel of figure 8.

The slight curve at high drift time in the reconstructed (S2) coordinates is due to the field transparency of the cathode, causing a leakage field from below the cathode [31]. In WS2013 this effect was corrected in the analysis without a need for a field map [27]. A similar effect caused by the transparency of the gate grid can be seen at low drift times near the gate grid. These field 

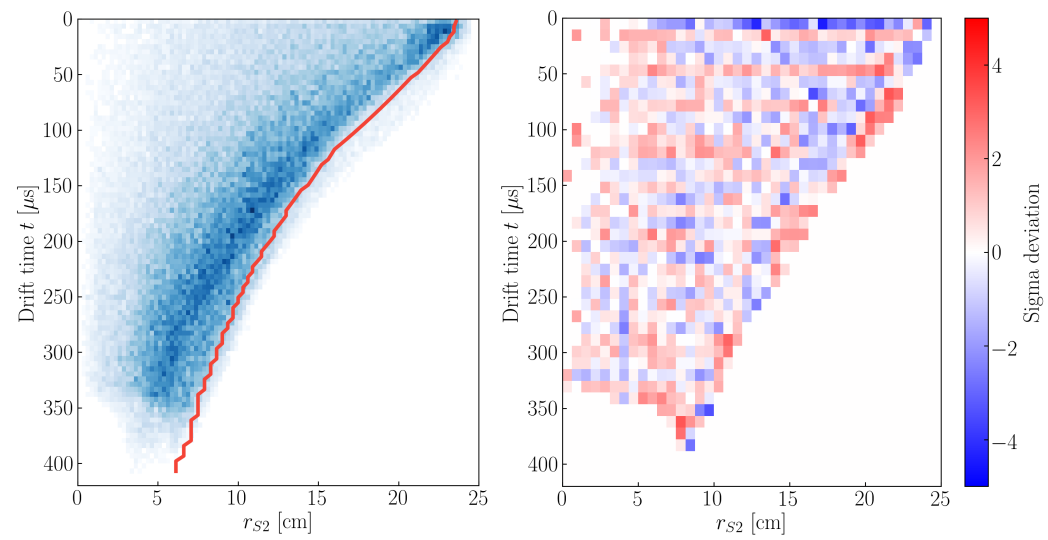

Figure 7. Left: solid red contour at $r=22 \mathrm{~cm}$ illustrates the modeled edge of the ${ }^{83 \mathrm{~m}} \mathrm{Kr}$ distribution (blue) from 2016-02-22. Right: variation between normalized histograms of ${ }^{83 \mathrm{~m}} \mathrm{Kr}$ data and simulation from 2016-10-06 weighted by the error in each bin (using an azimuthal slice between $120^{\circ} \leq \theta<180^{\circ}$ ).
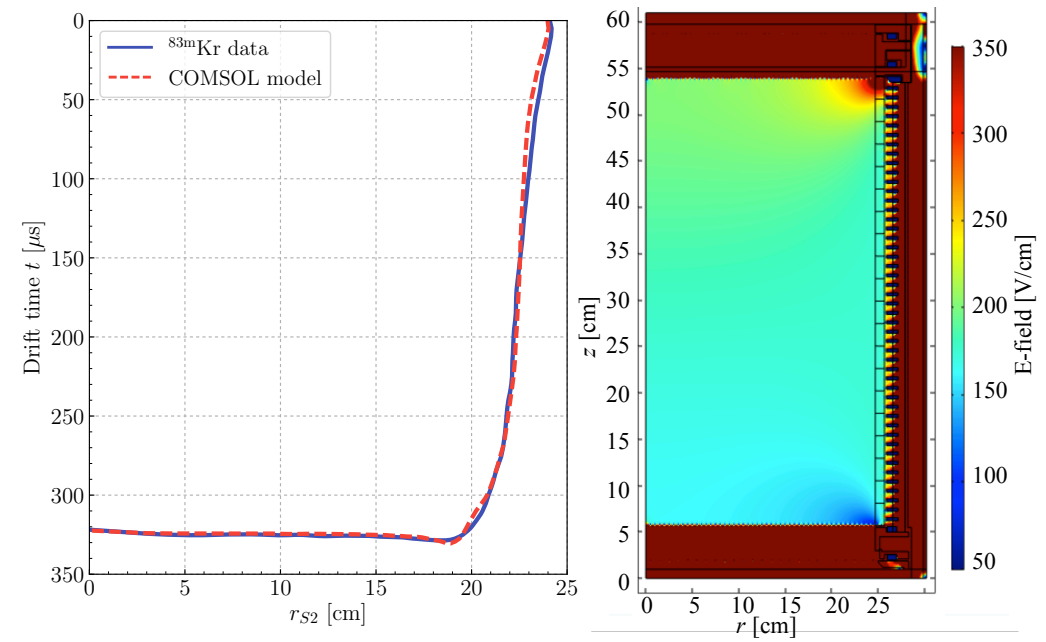

Figure 8. Left: contour plot showing histogram edges of the $3 \mathrm{D}$ field model and ${ }^{83 \mathrm{~m}} \mathrm{Kr}$ events averaged over all azimuthal angles during WS2013. Data (solid blue) and simulation (dashed red) contours were created following the method described in figure 1 without any fitting or tuning necessary. Right: analogous electric field strength in LUX during WS2013. Regions in the corners of the detector show field leakage through the transparent boundary grids.

leakages introduce a radial field component to the field lines as shown in figure 4, illustrating that an electron originating at high radius just above the cathode follows the field lines shown and reaches the liquid level at a radius reduced by several centimeters compared to the initial position.

Using the tiled charge model developed to study the electric field for WS2014-16, we were able to qualitatively improve our understanding of the WS2013 field model with respect to the detector wall shape. Even though the electric field in WS2013 was mostly uniform, a scalloping was seen along the edges of the detector that was not observed in the ideal COMSOL Multiphysics model 
without charge tiles, as shown in figure 9.

The same approach described in section 2.3 was used to model this scalloping effect in WS2013. Since the tiling was optimized for WS2014-16, the model was unable to fully reproduce all the fine features. The average fitted charge found in the PTFE panels was $-0.03 \mu \mathrm{C} / \mathrm{m}^{2}$, which is comparable to triboelectric charge densities. This illustrates the sensitivity of the field modeling method to capture even very small charge densities.

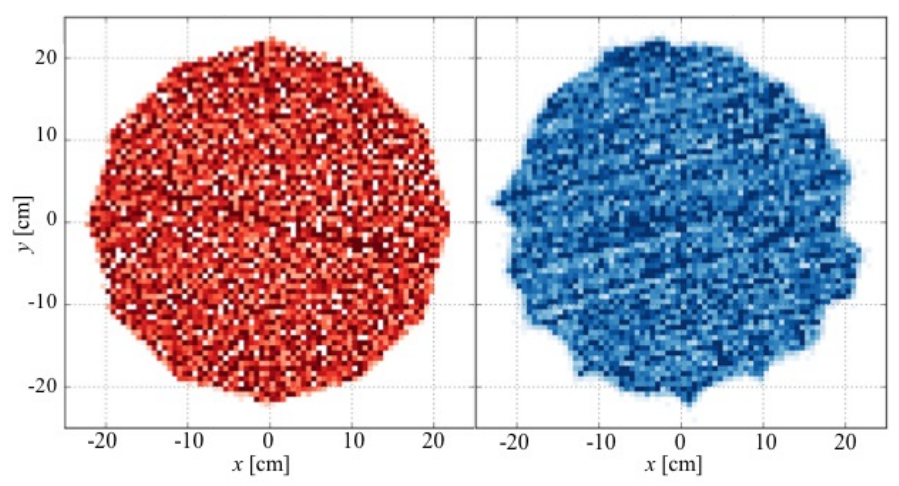

Figure 9. Left: original COMSOL model with no charge showing a clear dodecagonal cross-section. Right: data from the detector with visible scallops using ${ }^{83 \mathrm{~m}} \mathrm{Kr}$ data from 2013-05-10 during WS2013. Also visible is the orientation of gate wires. Both cross-sections are a slice at a drift time of $255 \mu \mathrm{s}<t \leq 290 \mu \mathrm{s}$.

\subsection{Alternative determination of electric field magnitude using NEST}

An alternative determination of the electric fields was done by fitting the field as a free parameter in the Noble Element Simulation Technique (NEST) package [32] to match calibration data. NEST encompasses the WIMP search community's understanding of both scintillation and ionization yields as well as the field impact on the electron-ion recombination probability. As the field magnitude is increased, the ionization signal increases while the scintillation signal decreases [33]. This is a result of the electric field influencing the fraction of electrons which recombine with ions at a particle-interaction site: if a given electron recombines with an ion, it contributes to the scintillation signal, otherwise it contributes to the ionization signal. NEST's underlying physics model accounts for these electron-ion recombination effects, and has been refined with recent measurements in the literature [34] to extend up to high fields.

Because NEST requires an electric field magnitude as input, the field value can be treated as a free parameter and NEST's output fit to calibration data. By performing such a fit to calibration data from a specific region of the detector, an estimate of the field magnitude in that region at the time of the calibration was obtained. This technique for estimating field is completely independent from the technique described in previous sections: this technique uses the size of the scintillation and ionization pulses, while the technique described previously in section 2 uses only the spatial distribution of the pulses.

Though ${ }^{83 \mathrm{~m}} \mathrm{Kr}$ decays were used for the field estimates in previous sections, this source is not conducive to analysis with NEST for this purpose because of its complicated LXe response [35] due to its decay [36], which is difficult to model. Instead, periodic calibrations with tritiated methane 
$\left(\mathrm{CH}_{3} \mathrm{~T}\right)$ dissolved in the LXe were used. The decay of this source is very simple and covers a wide range of energies [37]. Data collected from this source form a band in the space of $\log _{10}(\mathrm{~S} 2 / \mathrm{S} 1)$ vs. S1, whose mean and width depends on the applied field. The spatial variation in detector response was handled by dividing the fiducial volume into four regions of drift time; the boundaries of these regions were 40, 105, 170, 235, $300 \mu \mathrm{s}$.

$\mathrm{CH}_{3} \mathrm{~T}$ data were segregated into these four regions, and the band from each was considered independent. Four spatial regions were found to be the optimal number: more regions would result in too few calibration statistics with which to tune the model for each region, while fewer regions would not adequately capture the field variation in the full fiducial volume.

The $\mathrm{CH}_{3} \mathrm{~T}$ band from each drift-time region was broken up into bins of $\mathrm{S} 1$; for each $\mathrm{S} 1$ bin, the distribution of events in $\log _{10}(\mathrm{~S} 2 / \mathrm{S} 1)$ was fit with a normal distribution. The $\mathrm{CH}_{3} \mathrm{~T}$ band as a function of S1 was characterized by the means and widths of these fits, and these were compared to the means and widths predicted by the output of NEST. Two NEST parameters were varied until optimal agreement between NEST and $\mathrm{CH}_{3} \mathrm{~T}$ data was obtained; these parameters were (1) the applied field (which affects mainly the mean value of the electron-ion recombination fraction), and (2) a parameter, $f_{r}$ that characterizes the size of the fluctuations in this electron-ion recombination. Because the electric field is considered uniform within a given fiducial-volume region, residual variations in the field were empirically accounted for by increasing $f_{r}$. Figure 10 (left) shows the comparison between data and best-fit model for $\mathrm{CH}_{3} \mathrm{~T}$ in one of the four spatial regions at the beginning of the WIMP-search run. Data and best-fit models for all four drift-time regions, at all times during the WIMP-search run are consistent. The quantities in the axis labels are given as "S1c" and "S2c", which indicate that the pulse areas have been corrected for variations resulting from non-field-related effects such as xenon purity and geometrical optics.

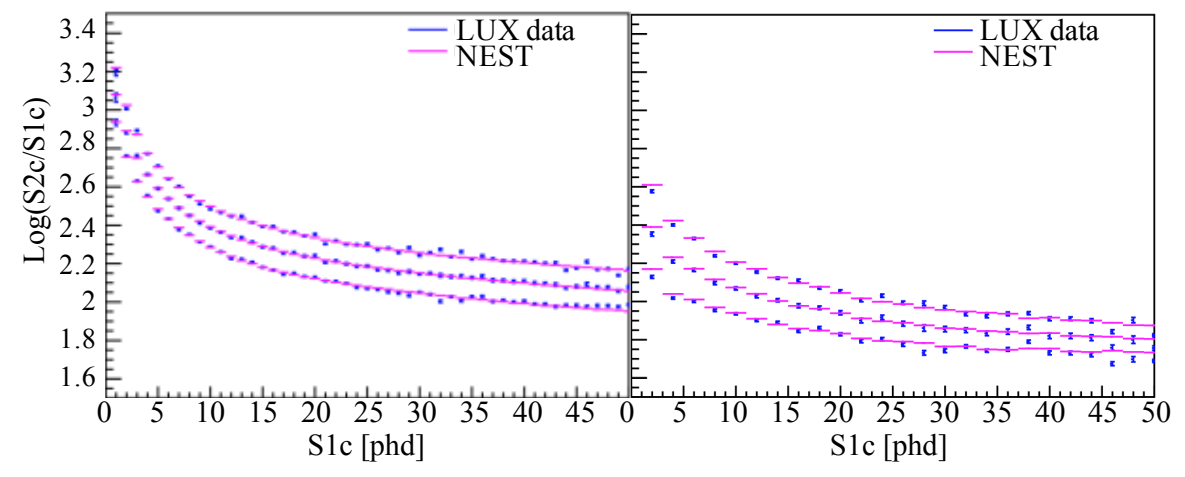

Figure 10. Agreement between LUX data and NEST simulation for the ER band (left) and NR band (right) along with the 10\% and 90\% confidence level bands. Data shown are from September 11, 2014 - January 1, 2015 period with drift time $40 \mu \mathrm{s}<t<105 \mu$ s as defined in [4].

Because LXe's response to nuclear recoils is significantly different than for electronic recoils, nuclear recoils require a separate detector-response model than is obtained from the $\mathrm{CH}_{3} \mathrm{~T}$ calibrations. For this purpose, data collected with a collimated beam from a deuterium-deuterium (DD) neutron generator were used [38]. However, LXe's nuclear-recoil response is nearly unaffected by applied field [33], and therefore the NEST models that are applied to the DD calibrations use the 
field magnitudes as determined by the corresponding $\mathrm{CH}_{3} \mathrm{~T}$ calibration of the same spatial region and date. The fits of the NEST model to DD data therefore vary only the parameter $f_{r}$. Figure 10 (right) shows the comparison between bands from DD data and best-fit model for the same spatial region and date as the $\mathrm{CH}_{3} \mathrm{~T}$ band seen in the left plot.

\section{Time-dependence of electric fields during WS2014-16}

\subsection{Charge evolution}

The results of the method described here were one field map for each month of WS2014-16, corresponding to 21 field maps in total. As discussed earlier, each field map was produced by adjusting the negative charge distribution in the PTFE panels and fitting the modeled electric field to the detector observables. One of the resulting charge distributions in the PTFE panels is shown in figure 11 and the evolution of charge over the course of WS2014-16 is demonstrated in figure 12. The varying charge results in a time dependent electric field, changing also in azimuth and $z$ as illustrated in figure 13. Most negative charge was found to be in the top $1 / 3$ of the detector throughout all of WS2014-16, possibly due to electrons in the PTFE panels being pulled toward the gate while holes were attracted to the cathode at a faster rate.

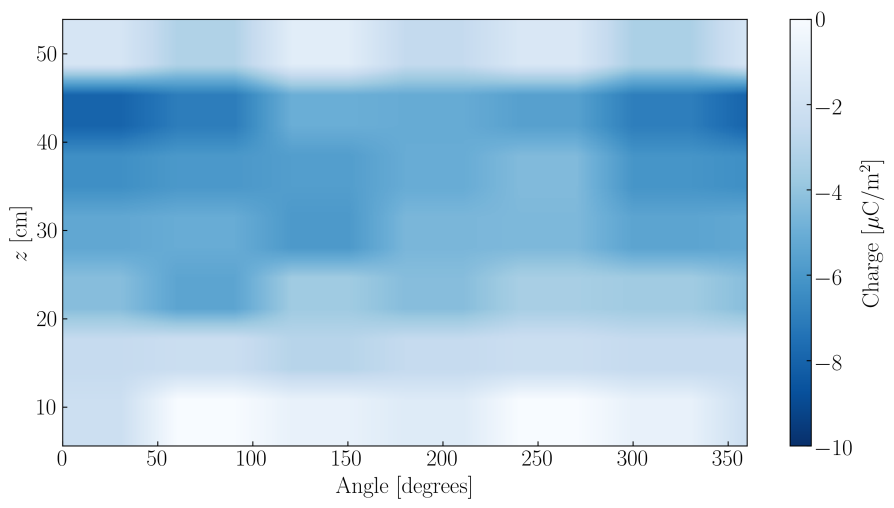

Figure 11. Charge density distribution in the detector's PTFE panels that resulted from fitting ${ }^{83 \mathrm{~m}} \mathrm{Kr}$ data from 2014-10-06. The 42 segments correspond to the smoothed tile charges as described in section 2.3.

The increasingly negative charge throughout WS2014-16 averaged over the entire PTFE area is plotted in figure 14. The cathode voltage was biased down to $0 \mathrm{~V}$ from April 7 to April 15, 2015 to investigate its effect on the charge in the PTFE panels. Data from this period was not used in analysis. After the cathode was re-biased to $-8.5 \mathrm{kV}$ the charge density was fit with an exponential function as in Reference [21]:

$$
\sigma=\sigma_{0} \exp (-t / \tau)+\sigma_{\infty}
$$

where $\sigma$ is the average surface charge density, $\sigma_{0}$ is the initial charge density, and $\sigma_{\infty}$ is the value of the charge $\lim _{t \rightarrow \infty} \sigma . \tau$ is the charge transit time. The best-fit values found were $\sigma_{0}=3.1 \mu \mathrm{C} / \mathrm{m}^{2}$, $\tau=181$ days, and $\sigma_{\infty}=-5.6 \mu \mathrm{C} / \mathrm{m}^{2}$. 
There is a lack of research of PTFE properties at LXe temperatures so it is difficult to compare this number to the literature due to different experimental conditions used, such as temperature or the electric field applied.

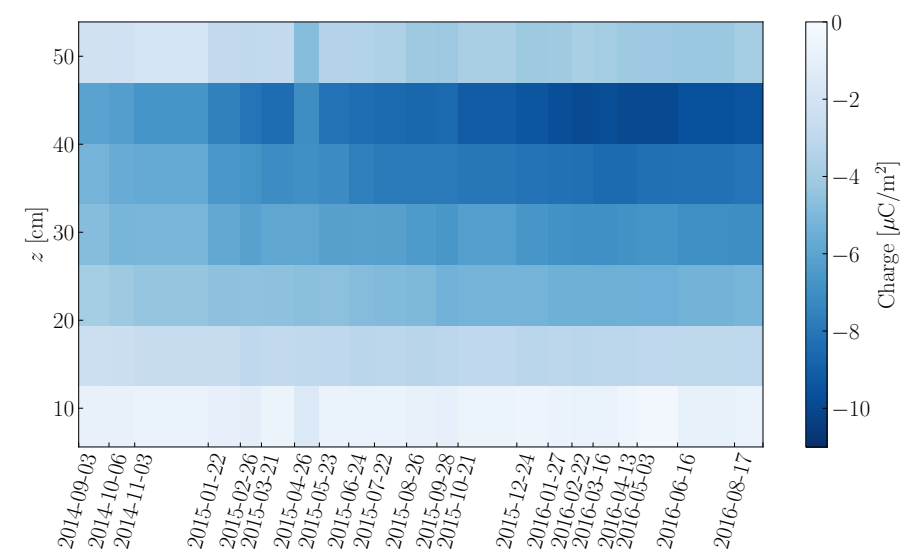

Figure 12. Charge density in each $z$ segment averaged over all azimuth throughout WS2014-16.
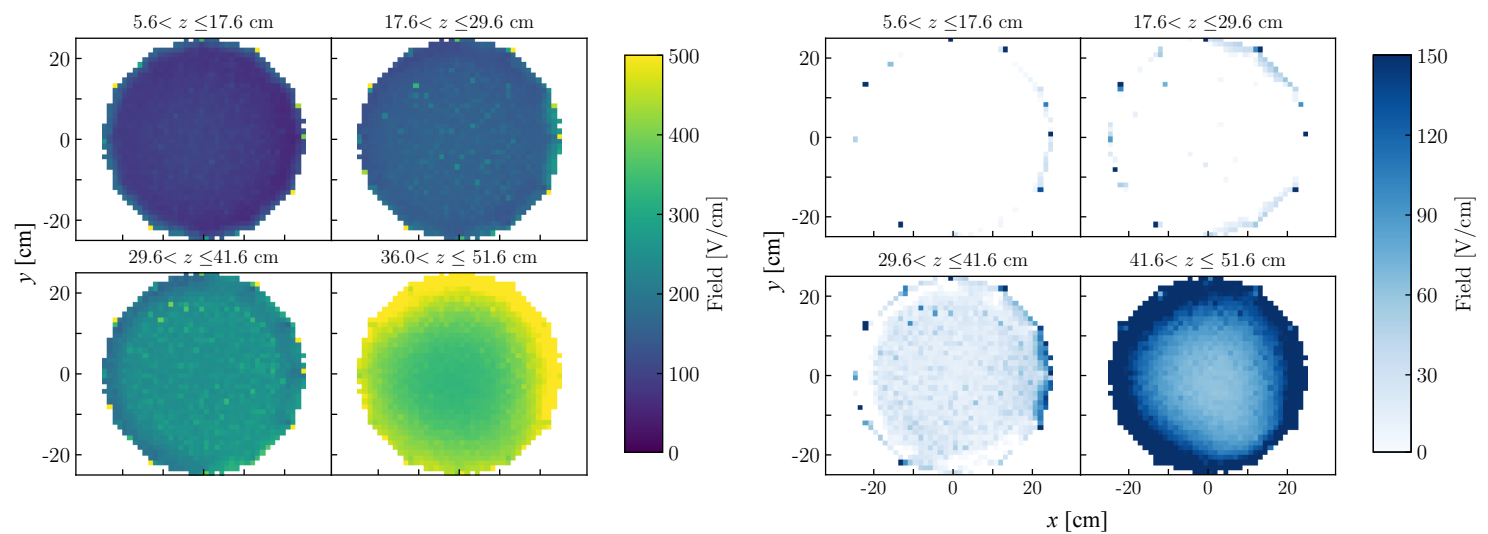

Figure 13. Left: electric field in different $z$ segments of the detector as modeled for 2014-09-03 dataset illustrating the azimuthal non-uniformity. Right: difference between the electric field at the end of WS201416 (field map from 2016-05-03) and the beginning (field map from 2014-09-03). The change in field is not uniform throughout WS2014-16.

\subsection{Electric fields in WS2014-16 analysis}

For simplicity of the analysis in [4] the data were divided into 16 bins, within each of which the change in the wall position was not significant. There were four bins in time, bounded by the following dates: September 11, 2014; January 1, 2015; April 1, 2015; October 1, 2015; May 2, 2016. Each of those bins was further split into four drift time bins with boundaries of 40, 105, 170, 235 , and $300 \mu$ s. Only one field map was chosen for each date bin, hence only four field maps were used in WS2014-16 analysis. Each map was chosen to be close to the average of the electric field 
values in the segment. The agreement between each field map and the given ${ }^{83 \mathrm{~m}} \mathrm{Kr}$ distribution for each date bin is shown in figure 15 .

Figure 16 shows the value of the electric field throughout the WS2014-16 analysis [4]. It shows the divergence of the field extrema as the electric field magnitude varied from $\sim 50-20 \mathrm{~V} / \mathrm{cm}$ near the bottom of the detector to $\sim 500-650 \mathrm{~V} / \mathrm{cm}$ near the top. The mean value of the field of $\sim 200 \mathrm{~V} / \mathrm{cm}$ remained mostly constant throughout the exposure.

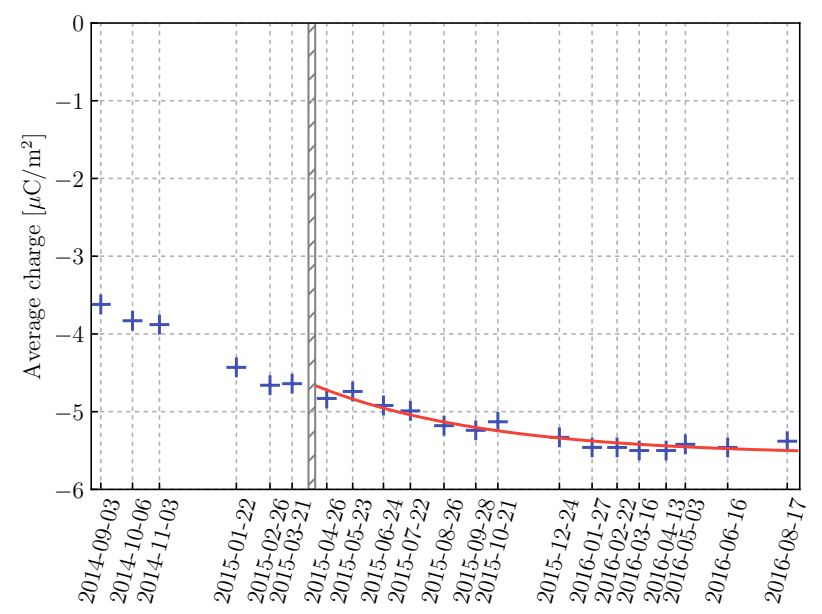

Figure 14. Increasingly negative charge density obtained from the field models, averaged over the entire PTFE panel surfaces for each month during WS2014-16. The data points correspond to the modeled average charge densities in the PTFE panels obtained from a small subset of the regular ${ }^{83 \mathrm{~m}} \mathrm{Kr}$ datasets. These data points can be fitted with an exponential function $\sigma=3.1 \exp (-t / 181)-5.6$ where $t$ is in units of "days since 2014-09-03". The gray dashed line indicates a week in April 2015 when the cathode voltage was biased down to $0 \mathrm{~V}$ to observe its effect on the charge.

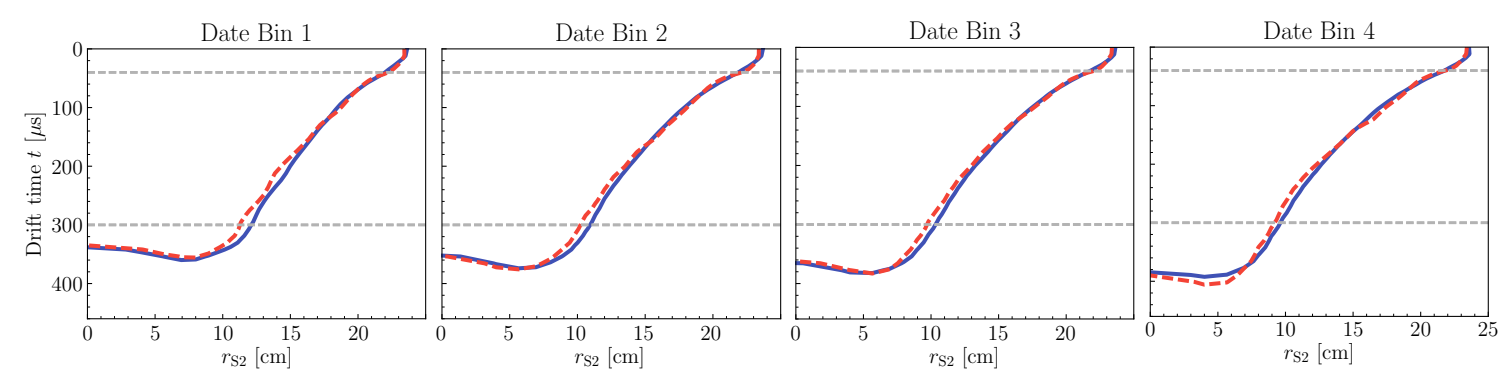

Figure 15. A comparison of the measured position of the detector wall and the cathode from ${ }^{83 \mathrm{~m}} \mathrm{Kr}$ (solid blue) to that predicted by the best-fit electric field model (dashed red). The contours were created following the method described in figure 1. Horizontal gray dashed lines, at 40 and $300 \mu \mathrm{s}$, indicate the drift time extent of the fiducial volume used in WS2014-16.

The volume-averaged fields obtained in COMSOL models can be compared to the NEST model fits that assume a uniform field within a given drift time bin. Figure 17 shows the comparison of the field magnitudes obtained by the NEST fits ("NEST Field") to the corresponding mean field values 

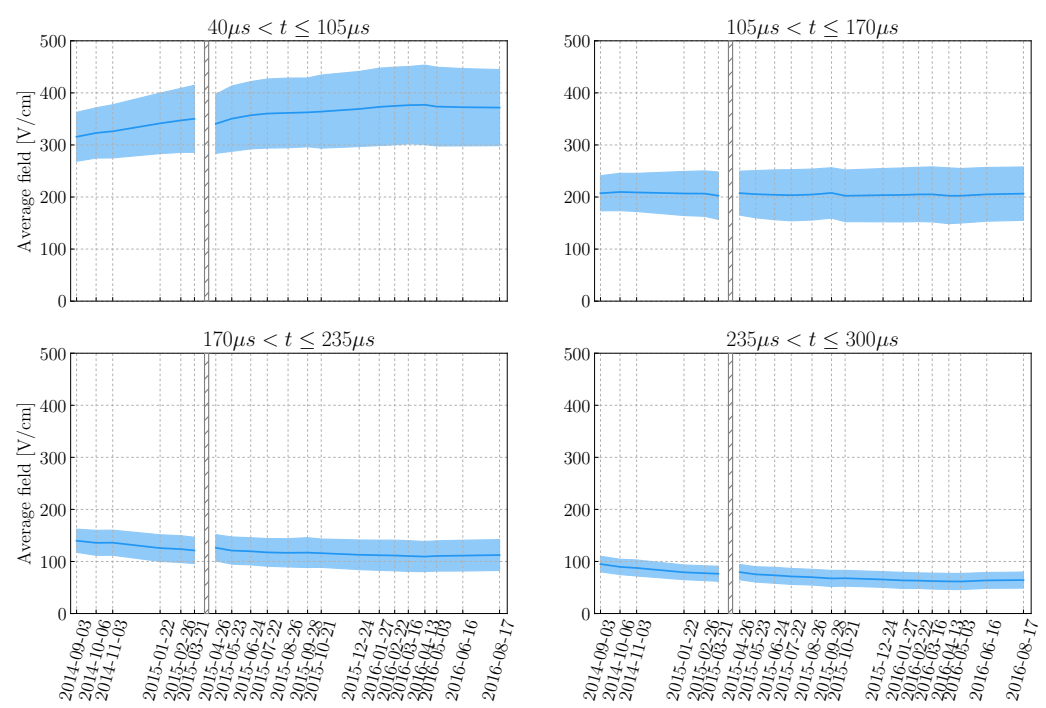

Figure 16. Average field during WS2014-16 in the four drift time bins used in analysis. Bands indicate the standard deviation of the field in the given drift time bin. A radial cut at $r=20 \mathrm{~cm}$ and a drift time cut at $40 \mu \mathrm{s}<d t \leq 300 \mu \mathrm{s}$ were applied. Gray dashed lines indicate a week in April 2015 when the cathode voltage was biased down to $0 \mathrm{~V}$.

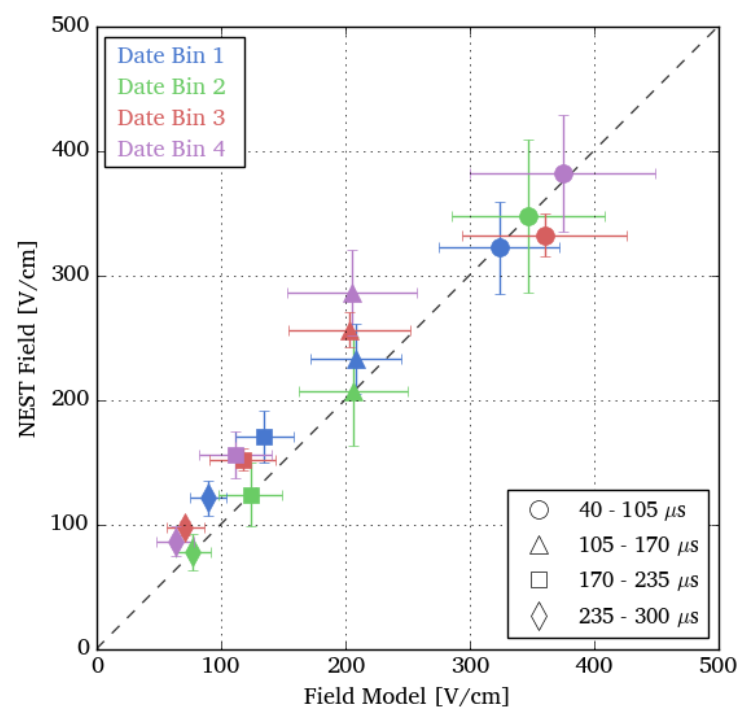

Figure 17. Comparison of electric field as modeled using the COMSOL modeling method described in section 2 to the best fit predicted by NEST for the 16 detector models (four spatial regions and four time periods) used in WS2014-16 analysis.

obtained from the COMSOL studies ("Field Model") for all 16 detector models (four spatial regions and four time periods). The field estimates obtained from the two methods show good agreement. This point deserves emphasis, because these two techniques for estimating electric field magnitude are completely independent: the electrostatic field model is based on the observed electron drift 
paths alone, while the NEST fits are based on the S1 and S2 amplitudes alone. Therefore we have confidence in the S1 and S2 signal reconstruction techniques used in the LUX WIMP search analysis.

\section{Summary}

A method was developed for detailed modeling of the varying electric fields inside the active volume of the LUX detector throughout its scientific operations. Understanding of the electric field was vital for the ER and NR signal reconstruction, the foundation of the LUX WIMP search analysis. The fields observed in the bulk of the active volume of the detector during WS2013 were reproduced without any tuning necessary. The COMSOL field map modeling method described in this work is sensitive down to $-0.03 \mu \mathrm{C} / \mathrm{m}^{2}$, enough charge density to model a minor scalloping to the detector edge as seen in WS2013.

The time dependence of the electric field during WS2014-16 is likely due to the creation of charge in the PTFE panels during detector grid conditioning prior to the extended run. The model of electric fields was created by simulating the LUX detector in 3D in COMSOL Multiphysics, where hypothetical charges were deposited in the PTFE panels surrounding the active region and varied until convergence on the distribution of ${ }^{83 \mathrm{~m}} \mathrm{Kr}$ calibration data within the detector was obtained. The resulting method allowed a faithful reconstruction of electric fields in the LUX detector and was critical for accurate event reconstruction and background discrimination during the WS2014-16 analysis [4]. The fields in WS2014-16 varied in time, azimuth and depth and the method described in this work was able to capture this evolution in all dimensions. Twenty-one field maps were developed to accurately model the changing electric field throughout the extended WS2014-16 with an average modeled charge distribution in the PTFE panels varying from -3.6 to $-5.5 \mu \mathrm{C} / \mathrm{m}^{2}$.

The mean electric field values resulting from this COMSOL modeling method were found to be in good agreement with electric field values deduced from the NEST package, which fit the field-dependent scintillation and ionization yields of the $\mathrm{CH}_{3} \mathrm{~T}$ calibration source. The successful modeling of electric fields, along with the strong calibration and simulation program developed by the LUX collaboration enabled a thorough understanding of the LUX detector throughout all of the scientific program and strengthened its sensitivity to WIMPs.

\section{Acknowledgments}

This work was partially supported by the U.S. Department of Energy (DOE) under Award No. DEAC02-05CH11231, No. DE-AC05-06OR23100, No. DE-AC52-07NA27344, No. DE-FG01-91ER40618, No. DE-FG02-08ER41549, No. DE-FG02-11ER41738, No. DE-FG02-91ER40674, No. DE-FG0291ER40688, No. DE-FG02-95ER40917, No. DE-NA0000979, No. DE-SC0006605, No. DESC0010010, and No. DE-SC0015535; the U.S. National Science Foundation under Grants No. PHY0750671, No. PHY-0801536, No. PHY-1003660, No. PHY-1004661, No. PHY-1102470, No. PHY1312561, No. PHY-1347449, No. PHY-1505868, and No. PHY-1636738; the Research Corporation Grant No. RA0350; the Center for Ultra-low Background Experiments in the Dakotas (CUBED); and the South Dakota School of Mines and Technology (SDSMT). LIP-Coimbra acknowledges funding 
from Fundação para a Ciência e a Tecnologia (FCT) through the Project-Grant No. PTDC/FISNUC/1525/2014. Imperial College and Brown University thank the UK Royal Society for travel funds under the International Exchange Scheme (No. IE120804). The UK groups acknowledge institutional support from Imperial College London, University College London and Edinburgh University, and from the Science \& Technology Facilities Council for PhD studentships No. ST/K502042/1 (AB), No. ST/K502406/1 (SS) and No. ST/M503538/1 (KY). The University of Edinburgh is a charitable body, registered in Scotland, with Registration No. SC005336.

This research was conducted using computational resources and services at the Center for Computation and Visualization, Brown University, and also the Yale Science Research Software Core. The ${ }^{83} \mathrm{Rb}$ used in this research to produce ${ }^{83 \mathrm{~m}} \mathrm{Kr}$ was supplied by the United States Department of Energy Office of Science by the Isotope Program in the Office of Nuclear Physics.

We would like to thank the referees for their constructive comments and recommendations. We gratefully acknowledge the logistical and technical support and the access to laboratory infrastructure provided to us by SURF and its personnel at Lead, South Dakota. SURF was developed by the South Dakota Science and Technology Authority, with an important philanthropic donation from T. Denny Sanford, and is operated by Lawrence Berkeley National Laboratory for the Department of Energy, Office of High Energy Physics.

\section{References}

[1] LUX collaboration, D. S. Akerib et al., First results from the LUX dark matter experiment at the Sanford Underground Research Facility, Phys. Rev. Lett. 112 (2014) 091303, [1310. 8214].

[2] LUX collaboration, D. S. Akerib et al., Improved Limits on Scattering of Weakly Interacting Massive Particles from Reanalysis of 2013 LUX Data, Phys. Rev. Lett. 116 (2016) 161301, [1512 . 03506$].$

[3] LUX collaboration, D. S. Akerib et al., Results on the Spin-Dependent Scattering of Weakly Interacting Massive Particles on Nucleons from the Run 3 Data of the LUX Experiment, Phys. Rev. Lett. 116 (2016) 161302, [1602.03489].

[4] LUX collaboration, D. S. Akerib et al., Results from a search for dark matter in the complete LUX exposure, Phys. Rev. Lett. 118 (2017) 021303, [1608.07648].

[5] LUX collaboration, D. S. Akerib et al., First searches for axions and axion-like particles with the LUX experiment, 1704.02297.

[6] LUX collaboration, D. S. Akerib et al., Limits on spin-dependent WIMP-nucleon cross section obtained from the complete LUX exposure, Phys. Rev. Lett. 118 (2017) 251302, [1705 . 03380].

[7] LUX collaboration, D. S. Akerib et al., The Large Underground Xenon (LUX) Experiment, Nucl. Instrum. Meth. A704 (2013) 111-126, [1211.3788].

[8] M. Anelli, P. Campana, E. Dane, C. Forti, G. Martellotti, G. Penso et al., Quality tests of the lhcb muon chambers at the lnf production site, IEEE Transactions on Nuclear Science 53 (Feb, 2006) 330-335.

[9] G. Arnison et al., Production and testing of limited streamer tubes for the end-cap muon subdetector of opal, Nuclear Instruments and Methods in Physics Research Section A: Accelerators, Spectrometers, Detectors and Associated Equipment 294 (1990) 431 - 438.

[10] J. P. DeWulf et al., Test results and conditioning procedure of a limited streamer-tube calorimeter, Nuclear Instruments and Methods in Physics Research Section A: Accelerators, Spectrometers, Detectors and Associated Equipment 263 (1988) 109 - 113. 
[11] A. Arefiev et al., Proportional chambers for the barrel hadron calorimeter of the 13 experiment, Nuclear Instruments and Methods in Physics Research Section A: Accelerators, Spectrometers, Detectors and Associated Equipment 275 (1989) 71 - 80.

[12] F. Neves, A. Lindote, A. Morozov, V. Solovov, C. Silva, P. Bras et al., Measurement of the absolute reflectance of polytetrafluoroethylene (PTFE) immersed in liquid xenon, JINST 12 (2017) P01017, [1612.07965].

[13] I. Kanik, Far ultraviolet emission spectrum of xenon induced by electron impact at 100 ev, Chemical Physics Letters 258 (1996) 455 - 459.

[14] A. J. P. L. Policarpo, Light production and gaseous detectors, Physica Scripta 23 (1981) 539.

[15] R. D. Andresen, E.-A. Leimann and A. Peacock, The nature of the light produced inside a gas scintillation proportional counter, Nuclear Instruments and Methods 140 (1977) 371 - 374.

[16] M. Yun, K. Yoshino, Y. Inuishi and M. Kawatsu, Photoconduction in polytetrafluoroethylene induced by vacuum-ultraviolet light, Japanese Journal of Applied Physics 21 (1982) 1592.

[17] K. Seki, H. Tanaka, T. Ohta, Y. Aoki, A. Imamura, H. Fujimoto et al., Electronic structure of poly(tetrafluoroethylene) studied by ups, vuv absorption, and band calculations, Physica Scripta 41 (1990) 167.

[18] G. J. Zhang, K. Yang, W. B. Zhao and Z. Yan, On the surface trapping parameters of polytetrafluoroethylene block, Applied Surface Science 253 (2006) 1995 - 1998.

[19] R. Hanna, T. Paulmier, P. Molinie, M. Belhaj, B. Dirassen, D. Payan et al., Radiation induced conductivity in space dielectric materials, Journal of Applied Physics 115 (2014) 033713, [http://dx.doi.org/10.1063/1.4862741].

[20] N. W. Green, A. R. Frederickson and J. R. Dennison, Experimentally derived resistivity for dielectric samples from the crres internal discharge monitor, IEEE Transactions on Plasma Science 34 (Oct, 2006) 1973-1978.

[21] A. Mellinger, Charge storage in electret polymers: mechanisms, characterization and applications. PhD thesis, University Potsdam, 2004.

[22] X. Zhang-Fu, D. Hai, Y. Guo-Mao, L. Ting-Ji and S. Xi-Min, Constant-current corona charging of teflon pfa, IEEE Transactions on Electrical Insulation 26 (Feb, 1991) 35-41.

[23] R. Kressmann, G. M. Sessler and P. Gunther, Space-charge electrets, IEEE Transactions on Dielectrics and Electrical Insulation 3 (Oct, 1996) 607-623.

[24] "COMSOL Multiphysics ${ }^{\circledR}$."

[25] K. T. McDonald, Notes on electrostatic wire grids, Internal note, available at http://WwW. hep . princeton. edu/ mcdonald/examples/grids.pdf (2003) .

[26] EXO-200 collaboration, J. B. Albert et al., Measurement of the Drift Velocity and Transverse Diffusion of Electrons in Liquid Xenon with the EXO-200 Detector, Phys. Rev. C95 (2017) 025502, [1609. 04467].

[27] LUX collaboration, D. S. Akerib et al., ${ }^{83 m} \mathrm{Kr}$ calibration of the 2013 LUX dark matter search, 1708.02566.

[28] S. Chib and E. Greenberg, Understanding the metropolis-hastings algorithm, The American Statistician 49 (1995) 327-335. 
[29] LUX collaboration, D. Akerib et al., Luxsim: A component-centric approach to low-background simulations, Nuclear Instruments and Methods in Physics Research Section A: Accelerators, Spectrometers, Detectors and Associated Equipment 675 (2012) 63 - 77.

[30] LUX collaboration, D. S. Akerib et al., "Backgrounds in the extended LUX rare event search.".

[31] Y. Mei, Direct Dark Matter Search with the XENON100 Experiment. PhD thesis, Rice University, 2012.

[32] M. Szydagis, A. Fyhrie, D. Thorngren and M. Tripathi, Enhancement of NEST Capabilities for Simulating Low-Energy Recoils in Liquid Xenon, JINST 8 (2013) C10003, [1307.6601].

[33] E. Aprile, C. E. Dahl, L. DeViveiros, R. Gaitskell, K. L. Giboni, J. Kwong et al., Simultaneous measurement of ionization and scintillation from nuclear recoils in liquid xenon as target for a dark matter experiment, Phys. Rev. Lett. 97 (2006) 081302, [astro-ph/0601552].

[34] Q. Lin, J. Fei, F. Gao, J. Hu, Y. Wei, X. Xiao et al., Scintillation and ionization responses of liquid xenon to low energy electronic and nuclear recoils at drift fields from $236 \mathrm{~V} / \mathrm{cm}$ to $3.93 \mathrm{kV} / \mathrm{cm}$, Phys. Rev. D92 (2015) 032005, [1505.00517].

[35] L. Baudis, H. Dujmovic, C. Geis, A. James, A. Kish, A. Manalaysay et al., Response of liquid xenon to Compton electrons down to $1.5 \mathrm{keV}$, Phys. Rev. D87 (2013) 115015, [1303.6891].

[36] A. Chan et al., Performance of the HPC calorimeter in DELPHI, IEEE Trans. Nucl. Sci. 42 (1995) $491-498$.

[37] LUX collaboration, D. S. Akerib et al., Tritium calibration of the LUX dark matter experiment, Phys. Rev. D93 (2016) 072009, [1512 .03133].

[38] LUX collaboration, D. S. Akerib et al., Low-energy $(0.7-74 \mathrm{keV})$ nuclear recoil calibration of the LUX dark matter experiment using D-D neutron scattering kinematics, 1608.05381. 